\title{
Compositional Analysis of Lentil (Lens culinaris) Cultivars Related to Colors and Their Antioxidative Activity
}

\author{
So-Young Lee ${ }^{1}$, Yun-Soo Yeo ${ }^{1}$, Soo-Yun Park ${ }^{1}$, Seong-Gon Lee ${ }^{1}$, Si-Myung Lee ${ }^{1}$, Hyun-Suk Cho ${ }^{1}$, Nam-Jin Chung ${ }^{2}$, \\ Seon-Woo $\mathrm{Oh}^{1} *$ \\ ${ }^{1}$ National Institute of Agricultural Science, Rural Development Administration, Jeonju 54874, Korea \\ ${ }^{2}$ Department of Crop Science and Biotechnology, Chonbuk National University, Jeonju 54896, Korea
}

\begin{abstract}
Metabolite profile is a powerful analytical technique to identify the functional characterization of plants. In this study, the phytochemicals and secondary metabolites of lentils (Lens culinaris) were analyzed to compare the anti-oxidative activities according to the different colors. The polar metabolites, fatty acids, carotenoids, flavonoids, anthocyanins, total phenolic acids, DPPH activity were analyzed. Three kind of lentils, French green whole lentil (FG), red whole lentil (LR), and green whole lentil (LG) (ASIA SEED Co., LTD), were used for this study. Fatty acids, phytochemicals, and antioxidative components from each lentil varieties were analyzed by official methods. The contents of lutein in carotenoids were 6-9 times higher than zeaxanthin in all lentils, but were not significantly different among three varieties. The content of carotenoids in FG was lower significantly than those in the LR and LG. Myricetin and luteolin were detected in the only FG. Kaempferol and delphinidin were significantly highest in the FG. Most of the phenolic acids except coumarate were higher in FG and LG than in LR. Also antioxidant effects (EC $\left.\mathrm{E}_{50}\right)$ were higher in FG and LG than in LR. The analyzed metabolites obtained from lentils showed distinct separation in the PCA results according to the varieties. Also, lentils showed different anti-oxidant profiles according to the colors. FG and LG showing higher contents of phytochemicals showed higher antioxidative activity than LG containing relative low contents of phytochemicals.
\end{abstract}

Keywords Lentil, Composition, Anthocyanin, Flavonoid, Antioxidant

\section{INTRODUCTION}

Legumes are high-quality and low-cost foods that can replace animal proteins (Mira et al. 2009), of which lentil (Lens culinaris) has the longest history of cultivation and is one of the five healthiest foods in the world (Xu et al. 2010). Lentils have since long been recognized as functional foods that promote health and have therapeutic properties such as antioxidant, anticancer, antibacterial activities, antihypertensive, immune regulatory, and cholesterol reducing properties (Shahidi and Zhong 2008; Nithiyanantham et al. 2012). In addition, lentils have a lower fat content than most legumes and as such, can be helpful for weight loss (Dueñas et al. 2002). In Korea, lentils were imported for the healthy functional food and their amount of import was about 12,000 ton in 2014. The import of lentils in 2014 increased 40 fold over 2013 (Korea custom services, trade statistics). Several kinds of lentils are imported in Korea such as red lentil (India/ Canada), brown lentil whole (India/Canada), red lentil whole (Australia), green lentil whole (India), French green lentil whole (Canada), light green lentil whole (Canada), Queen green lentil whole (Canada). Carotenoids are tetraterpenoid, fat-soluble pigments that can be subdivided into two groups based on whether or not they contain oxygen: lutein, zeaxanthin, and violaxanthin do contain

Received June 16, 2017; Revised August 3, 2017; Accepted August 4, 2017; Published September 1, 2017

*Corresponding author Seon-Woo Oh, ohsw0507@korea.kr, Tel: +82-63-238-4709, Fax: +82-63-238-4704 
oxygen, and $\alpha$-carotene, $\beta$-carotene, and lycopene do not contain it (Bouvier et al. 2005; Bhakta and Siva 2010; Cazzonelli 2011). In contrast, flavonoids, the largest group of secondary metabolites (Kim et al. 2013), which have been studied in snapdragon, corn, Arabidopsis, and petunia (Jaakola and Hohtola 2010), are water-soluble pigments. In addition, approximately 20 types of anthocyanins, flavonoid-type pigments producing purple, red, and blue colors, are found in plants (Harborne 1993). They act as antioxidants - bioactive compounds that delay ageing and hamper disease onset in humans and prevent food degradation by oxidation (Kim et al. 2014). Kim et al. (2006) reported that differences in anti-oxidative activity among legume varieties, as measured by their ability to scavenge 2,2-diphenyl-1-picylhydrazyl (DPPH) free radicals, were highly correlated with anthocyanin contents in the seed coats. Metabolomics is a useful tool for determining phenotypic variation in biological samples (Steuer 2006). This study was conducted to identify and compare the profiles of phytochemicals and secondary metabolites in the lentils with different colors. We profiled the abundant hydrophilics using GC-TOF MS, and quantified carotenoids and anthocyanins using HPLC of three different lentils. The analyzed components were fatty acids as main nutrients, polar metabolites as secondary metabolites and phenotype-related metabolites and their derivative secondary metabolites. Finally, the contents of nutrients were compared with each other by investigating relationships between phenotype-related metabolites and their derivative secondary metabolites. The nutrient profiles of lentils were also showed with chemometrics using principal component analysis (PCA) and hierarchical clustering analysis (HCA) form data calculating by Pearson's correlation to determine the relationships between color and metabolites.

\section{MATERIALS AND METHODS}

\section{Materials}

The three kind of lentils used in this study were commercial varieties which were imported from America and had the biggest market share. These were French green whole lentil (FG), red whole lentil (LR), and green whole lentil (LG) (Fig. 1). These samples were purchased from ASIA SEED Co., LTD. Fresh samples were freeze-dried for at least 72 hours and then crushed to a fine powder with the Planetary Mono Mill (Pulverisette 6; Fritsch, IdarOberstein, Germany). The powder was stored at $-80^{\circ} \mathrm{C}$ before analysis.

\section{Polar metabolites analysis}

Polar metabolites were extracted from $0.1 \mathrm{~g}$ samples using $1 \mathrm{~mL}$ of $2.5: 1: 1 \mathrm{v} / \mathrm{v} / \mathrm{v}$ methanol/water/chloroform. Ribitol $(0.06 \mathrm{~mL}, 0.2 \mathrm{mg} / \mathrm{mL}$ of water) was added as an internal standard (IS). The extraction was performed at $37^{\circ} \mathrm{C}$ with a mixing frequency of $1,200 \mathrm{rpm}$ for 30 minutes using a Thermomixer (compact model 5355; Eppendorf AG, Hamburg, Germany). The solutions were centrifuged at $16,000 \times g$ for $3 \mathrm{~min}$. The polar phase $(0.8 \mathrm{~mL})$ was transferred into a new tube, $0.4 \mathrm{~mL}$ of water was added, and the solution was centrifuged at $16,000 \times g$ for 3 minutes.
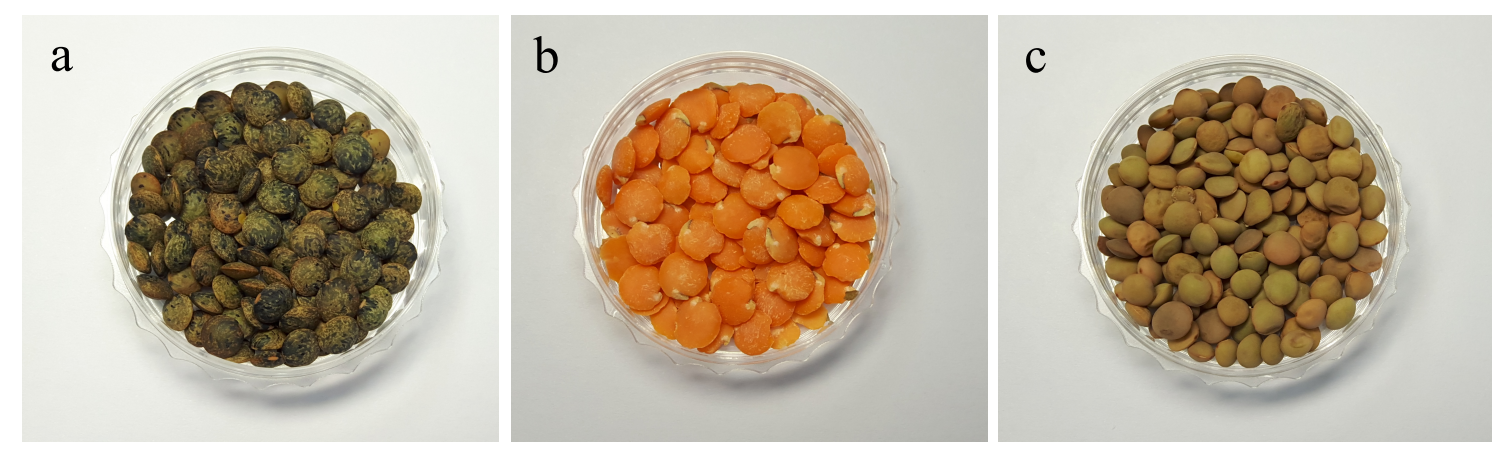

Fig. 1. Lentil samples used in this study. a: French green whole lentil (FG), b: red whole lentil (LR), c: green whole lentil (LG). 
The methanol/water phase was dried in a centrifugal concentrator (CVE-2000; Eyela, Japan) for $2.5 \mathrm{~h}$ and then freeze-dried for $15 \mathrm{~h}$. For oximation, the samples were dissolved in methoxyamine hydrochloride $(20 \mathrm{mg} / \mathrm{mL}$, $0.08 \mathrm{~mL}$ ) and incubated at $30^{\circ} \mathrm{C}$ for 90 minutes. Then, trimethylsilyl etherification was carried out by adding $0.08 \mathrm{~mL}$ of $N$-methyl- $N$-trimethylsilyl-trifluoroacetamide (MSTFA) and incubation at $37^{\circ} \mathrm{C}$ for 30 minutes. GC-TOF MS was carried out using an Agilent 7890A gas chromatograph (Agilent, Atlanta, GA, USA) coupled to a Pegasus HT TOF mass spectrometer (LECO, St. Joseph, MI, USA).

\section{Fatty acids analysis}

The fatty acid composition of the lipophilic fraction was analyzed according to the method described by AOCS (1997). The fatty acid content was determined using lipid extraction and saponification with $0.5 \mathrm{~N}$ sodium hydroxide in methanol. The saponification mixture was methylated with $14 \%$ boron trifluoride/methanol and the resulting methyl esters were extracted with pentane. Methyl esters of the fatty acids were analysed by GC (GC 7890B; Agilent, Atlanta, GA, USA). Pentadecanoic acid $(1 \mathrm{mg} / \mathrm{mL})$ was used as an IS.

\section{Carotenoid analysis}

Carotenoids were extracted from $0.1 \mathrm{~g}$ lentil seed samples by adding $3 \mathrm{~mL}$ of ethanol containing $0.1 \%$ ascorbic acid (w/v), vortex mixing, and incubation in a water bath at $85^{\circ} \mathrm{C}$ for 5 minutes. The carotenoid extract was saponified with potassium hydroxide $(0.12 \mathrm{~mL}, 80 \%$ $\mathrm{w} / \mathrm{v}$ ) in a water bath at $85^{\circ} \mathrm{C}$ for 10 minutes. After saponification, the samples were immediately placed on ice, and cold deionized water $(1.5 \mathrm{~mL})$ was added. $\beta$-Apo-8'-carotenal $(0.2 \mathrm{~mL}, 25 \mu \mathrm{g} / \mathrm{mL})$ was added as an IS. To separate the layers, carotenoids were extracted twice with hexane $(1.5 \mathrm{~mL})$ by centrifugation at $1,200 \times g$. Aliquots of the extracts were dried under a stream of nitrogen and re-dissolved in $50: 50(\mathrm{v} / \mathrm{v})$ dichloromethane/ methanol before HPLC analysis. The carotenoids were separated on a C30 YMC column $(250 \times 4.6 \mathrm{~mm}, 3 \mu \mathrm{m}$; YMC Co., Kyoto, Japan) on an Agilent 1100 HPLC instrument (Massy, France) equipped with a photodiode array detector. Chromatograms were generated at $450 \mathrm{~nm}$. Solvent A consisted of methanol/water $(92: 8 \mathrm{v} / \mathrm{v})$ with 10 $\mathrm{mM}$ ammonium acetate; solvent B consisted of $100 \%$ methyl tert-butyl ether. Solvent mobile phase was set with gradient program and the UV wavelength was $450 \mathrm{~nm}$.

\section{Flavonoid analysis}

Flavonoids were extracted from $0.1 \mathrm{~g}$ of lentil seed powder by adding $1.2 \mathrm{~mL}$ of $50 \%$ methanol containing 1.2 $\mathrm{M} \mathrm{HCl}$ in a water bath at $80^{\circ} \mathrm{C}$ for 2 hours. The crude suspensions were centrifuged at $10,000 \times g$ at $4^{\circ} \mathrm{C}$ for 5 minutes. The extracts were passed through $0.22-\mu \mathrm{m}$ Teflon polytetrafluoroethylene syringe filters and injected directly into the HPLC column. Flavonoid aglycones were separated on a $\mathrm{C}_{18}$ column $(250 \times 4.6 \mathrm{~mm}, 5 \mu \mathrm{m}$, Inertsil ODS-3; GL Sciences, Tokyo, Japan) on an HPLC instrument (Shimadzu, Kyoto, Japan) equipped with a photodiode array detector. Elution was performed using a binary gradient of $0.1 \%$ formic acid in water (mobile phase A) and $0.1 \%$ formic acid in acetonitrile (mobile phase B). Solvent mobile phase was set with gradient program and the PDA wavelength was $362 \mathrm{~nm}$.

\section{Anthocyanin analysis}

Anthocyanin extraction was performed according to a slightly modified method of Kim et al. (2010). The samples $(0.01 \mathrm{~g})$ were extracted with $0.95 \mathrm{~mL}$ of $85 \%$ methanol acidified with $1.0 \mathrm{~N} \mathrm{HCl}$ followed by sonication for 1 minute. The extracts were incubated at $38^{\circ} \mathrm{C}$ for 5 minutes with a mixing frequency of $500 \mathrm{rpm}$ using a Thermomixer comfort. The crude suspension was centrifuged at 10,000 $\times g$ at $4^{\circ} \mathrm{C}$ for 5 minutes, and the crude extract was hydrolysed with $0.2 \mathrm{~mL} 6 \mathrm{~N} \mathrm{HCl}$ at $90^{\circ} \mathrm{C}$ for 2 hours. After centrifugation, the hydrolysate was passed through a 0.22 $\mu \mathrm{m}$ polytetrafluoroethylene syringe filter prior to HPLC analysis. Anthocyanin was separated on a $\mathrm{C}_{18}$ column ( 250 $\times 4.6 \mathrm{~mm}, 5 \mathrm{um}$, Inertsil ODS-3, GL Sciences, Tokyo, Japan) as described above. Elution was performed using a binary gradient of $0.1 \%$ formic acid in water (mobile phase A) and $0.1 \%$ formic acid in acetonitrile (mobile phase B). Solvent mobile phase was set with gradient program and the PDA wavelength was $520 \mathrm{~nm}$. 


\section{Total phenolic acids analysis}

Soluble (free and esterified forms) and insoluble (bound form) phenolic acids were extracted according to the procedure described by Mira et al. (2009) with slight modifications. The powdered samples $(0.05 \mathrm{~g})$ were extracted twice by water-based sonication for $5 \mathrm{~min}$ at room temperature and incubation at $30^{\circ} \mathrm{C}$ for 10 minutes with $1 \mathrm{~mL}$ of $85 \%$ methanol containing $2 \mathrm{~g} / \mathrm{L}$ butylated hydroxyl anisole. After centrifugation at 13,000 rpm for 10 minutes at $4^{\circ} \mathrm{C}$, the combined extracts and residue were analyzed to determine the soluble and insoluble phenolic acid, respectively. 3,4,5-trimethoxycinnamic acid (100 $\mu \mathrm{g} / \mathrm{mL}$ ) was added as an IS, and the mixture was hydrolysed with $1 \mathrm{~mL}$ of $5 \mathrm{~N} \mathrm{NaOH}$ at $30^{\circ} \mathrm{C}$ under nitrogen gas for 4 hours. Each hydrolysed sample was adjusted to pH 1.5-2.0 with $6 \mathrm{M} \mathrm{HCl}$, extracted with ethyl acetate, and evaporated in a centrifugal concentrator (Eyela, Tokyo, Japan). For derivatisation, $40 \mu \mathrm{L}$ of MTBSTFA containing $1 \%$ TBDMCS and $40 \mu \mathrm{L}$ of pyridine were added to the dried extracts followed by incubation at $60^{\circ} \mathrm{C}$ for 30 minutes at a mixing frequency of 1,200 rpm using a Thermomixer comfort (Eppendorf AG, Hamburg, Germany). Each derivatised sample $(1 \mu \mathrm{L})$ was injected into a $7890 \mathrm{~A}$ gas chromatograph (Agilent, Atlanta, GA, USA) using a 7683A autosampler (Agilent). The column effluent was introduced into a Pegasus HT TOF mass spectrometer. The temperature was set with gradient program such as initial temperature $150^{\circ} \mathrm{C}(2$ minutes $), 320^{\circ} \mathrm{C}\left(15^{\circ} \mathrm{C} /\right.$ minute), and $320^{\circ} \mathrm{C}$ (10 minute holding).

\section{DPPH radical scavenging activity assay}

The powdered samples $(0.01 \mathrm{~g})$ were mixed with $1 \mathrm{~mL}$ of methanol and incubated at $30^{\circ} \mathrm{C}$ for 30 minutes at a mixing frequency of 1,200 rpm using a thermomixer comfort. After incubation, the supernatants were centrifuged at $10,000 \mathrm{rpm}$ at $4^{\circ} \mathrm{C}$ for 10 minutes. and then, $1 \mathrm{~mL}$ of methanol was added. This procedure was repeated. An extract dilution series of 25,50 , and $100 \%$ was made in methanol and pure methanol $(1 \mathrm{~mL})$ was used as a control. To compare the levels of scavenging activity, a dilution series of 2,6-di-tert-butyl-4-methylphenol (BHT) $(2.5,5$, 25,50 , and $100 \%$ ) was prepared. The extracts were reacted in dark conditions for 30 minutes by adding methanol $(1 \mathrm{~mL})$ containing $0.008 \%$ DPPH (2,2-diphenyl-1-picrylhydrazyl) and read out using a spectrophotometer (Beckman, DU650, California, USA) at $517 \mathrm{~nm}$ wavelength.

\section{Statistical analysis}

All determinations were repeated five times for reproducibility. Experimental data were analysed by the Duncan multiple-range test (SAS 9.2; SAS Institute, Cary, NC, USA) and analysis of variance (ANOVA). Principal component analysis (PCA) models for each components were developed by using for soft independent modeling of class analogy (BioPAT-SIMCA, ver. 13_Umetrics, Umeå, Sweden). Technically, a principal component was defined as a linear combination of optimally-weighted observed variables. In the course of performing a principal component analysis, it is possible to calculate a score for each subject on a given principal component. The PCA output consisted of score plots to visualize the contrast between different samples. The data file was scaled using unit variance scaling before all variables were subjected to PCA. Pearson correlation analysis was conducted with the SAS 9.2 software package (SAS Institute). Correlation analysis was conducted on the relative levels of 73 metabolites with standardization pre-processing. HCA and heat-map visualization of the correlation coefficient were performed using MultiExperiment Viewer version 4.4.0 software (http://www.tm4.org/mev/).

\section{RESULTS}

\section{Polar metabolites}

Hydrophilics with low molecular weight such as amino acids, organic acids, sugars, and sugar alcohols were analyzed by using GC-TOF MS. 42 peaks of untargeted metabolomics were identified through comparisons with related compounds in the library database of the Chroma TOF software (Fig. 2a). Relationships between metabolites were identified through Pearson correlation analysis and HCA. Metabolites were quantified based on the peak area ratio to an IS. The internal standard signal intensities obtained from the 42 metabolites were normalized based 

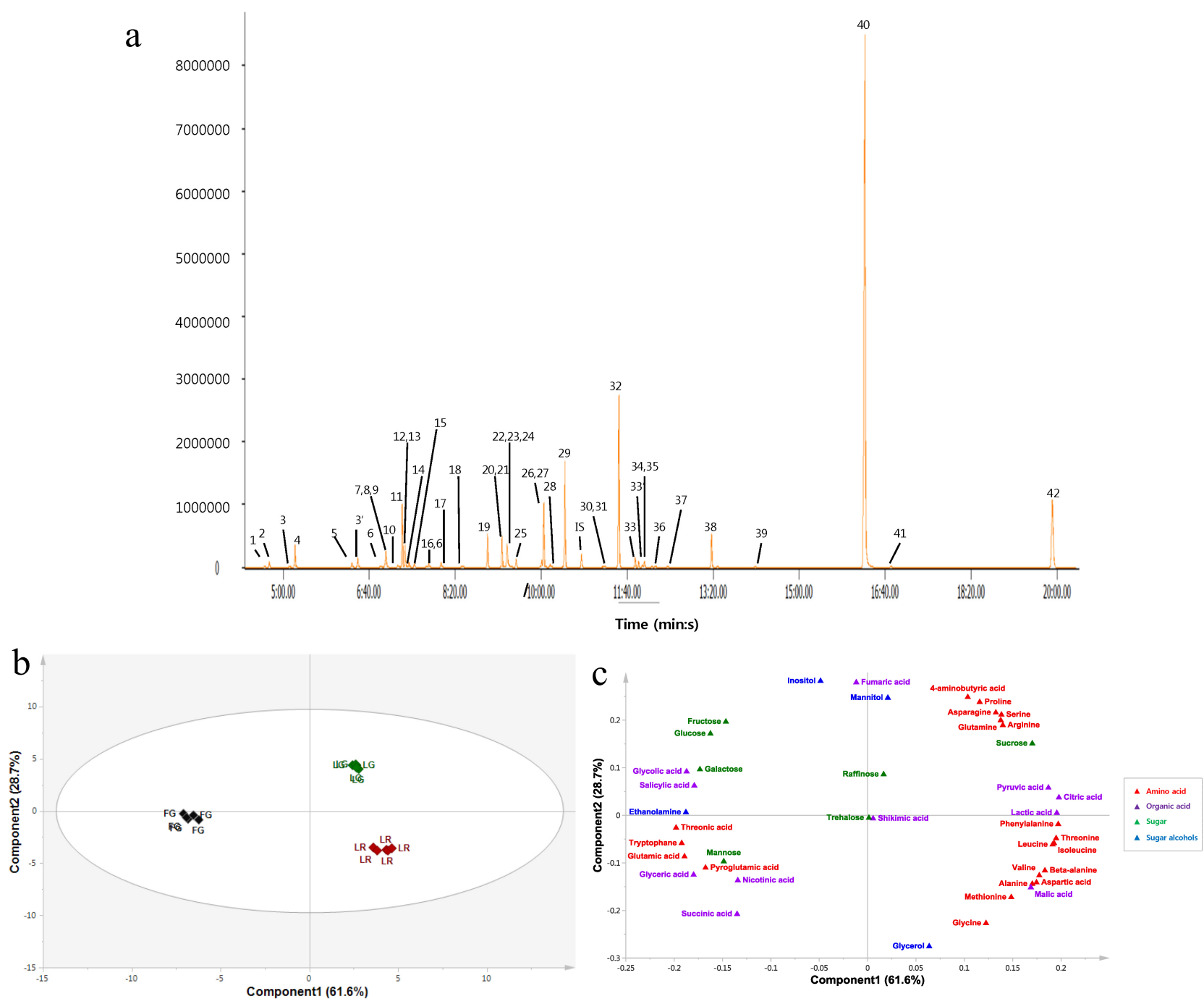

Fig. 2. Metabolite compounds from lentil seeds as determined by GC TOF-MS. (a) Selected ion chromatograms of hydrophilic metabolites extracted from LG lentil as MO/trimethylsilyl derivatives separated on a $30 \mathrm{~m} \times 0.25$ mmi. d. Fused silica capillary column coated with $0.25 \mu \mathrm{m}$ CP-SIL 8 CB low bleed. Peak identification: 1 , pyruvic acid; 2, lactic acid; 3, valine; 4, alanine; 5, glycolic acid; 6, serine; 7, ethanolamine; 8, glycerol; 9, leucine; 10, isoleucine; 11, proline; 12, nicotinic acid; 13, glycine; 14, succinic acid; 15, glyceric acid; 16, fumaric acid; 17, threonine; 18, beta-alanine; 19, malic acid; 20, salicylic acid; 21, aspartic acid; 22, methionine; 23, pyroglutamic acid; 24, 4-aminobutyric acid; 25, threonic acid; 26, arginine; 27, glutamic acid; 28, phenylalanine; 29, asparagine; 30, glutamine; 31, shikimic acid; 32, citric acid; 33, fructose; 34, galactose; 35, glucose; 36, Mannose; 37, mannitol; 38, Inositol; 39, tryptophane; 40, sucrose; 41, trehalose; 42, raffinose; IS, internal standard (ribitol). b-c: Score (b) and loading plots (c) of PC1 and PC2 of the PCA for hydrophilic data obtained from seeds of the three lentil varieties.

on PCA analysis to determine the variation in metabolite profiles between the cultivars. PCA models from data of polar metabolites of the three lentil varieties were to assess whether the lentil varieties showed separation according to the different color with different metabolite profiles. PCA score plots showed a distinct separation by different colored variety and the total deviation value of components $1(61.6 \%)$ and 2 (28.7\%) was 90.3\% (Fig. 2b). The identification and profiling of primary metabolites using GC-TOFMS analysis allows clear discrimination between 
lentil genotypes. The loading plot of 42 components ( 20 amino acids, 7 sugars, 4 sugar alcohols and 11 organic acids) was shown in Fig. 2c. PCA scores of shikimic acid of aromatic amino acid and trehalose of disaccharide were close to 0 showing independence. In addition, six amino acids (4-aminobutyric acid, proline, serine, asparagine, glutamine, and arginine) showed positive PCA score values, whereas three organic acids (glyceric acid, nicotinic acid, and succinic acid) and four amino acids (threonic acid, tryptophan, glutamic acid, and pyroglumic acid) had negative values. Even though they were present in small amounts, shikimic acid and trehalose were identified only in FG and LG, respectively, whereas mannose was detected only in FG and LG. From these results, metabolite profiles could be affected to the color characterization showing clear separation in the PCA chemometrics.

\section{Fatty acids}

Compared to other legumes, lentils are low in fat; therefore, it has been used as a plant protein source in weight-loss diets (Dueñas et al. 2002; Oh et al. 2009). The present study identified contents of saturated fatty acids including myristic acid (C14:0), palmitic acid (C16:0), and stearic acid (C18:0) and unsaturated fatty acids including oleic acid (C18:1), linoleic acid (C18:2), and linolenic acid (C18:3) (Table 1). The saturated fatty acid contents (C14:0, $\mathrm{C} 16: 0, \mathrm{C} 18: 0)$ were as low as $<10 \%$ of total fats. The unsaturated fatty acids made up the major part $( \pm 90 \%$ of total fatty acids), with linoleic acid (C18:2) being the most prevalent, consistent with the findings of Zhang et al. (2014) in 20 Canadian lentil cultivars. Similar results have been reported for sesame by Kang et al. (2000) and for soybean by Yoon et al. (1984) and Kim et al. (2007). In these studies, unsaturated fatty acids were also dominant and made up to $80 \%$ of total fatty acids. There were significant differences between LR and LG for stearic acid, among FG, LR, LG for oleic acid, between LR and LG for linoleic acid, and between FG and LR or LG for linolenic acid $(P<0.05)$. All variability among the treatments for the individual lentil components was explained by PC1 (component 1) and PC2 (component 2). The first components (PC1) of the PCA accounted for approximately $52.0 \%$ and PC2 was $20.3 \%$, respectively. The PCA obtained from the data of fatty acids showed clear separation between lentil varieties (Fig. 3a). These results

Table 1. Fatty acids, carotenoid, anthocyanin and flavonoid contents (mg/g DW) in seeds of three lentil varieties.

\begin{tabular}{llrrr}
\hline \hline & & $\mathrm{FG}^{\mathrm{z}}$ & $\mathrm{LR}^{\mathrm{z})}$ & $\mathrm{LG}^{\mathrm{z})}$ \\
\hline Fatty acids & Myristic acid (C14:0) & $0.14 \pm 0.02(0.70 \%) \mathrm{a}$ & $0.13 \pm 0.02(0.59 \%) \mathrm{a}$ & $0.11 \pm 0.03(0.62 \%) \mathrm{a}$ \\
& Palmitic acid (C16:0) & $1.25 \pm 0.03(6.24 \%) \mathrm{a}$ & $1.25 \pm 0.01(5.54 \%) \mathrm{a}$ & $1.27 \pm 0.02(7.19 \%) \mathrm{a}$ \\
& Stearic acid (C18:0) & $0.64 \pm 0.06(3.20 \%) \mathrm{b}$ & $0.66 \pm 0.04(2.90 \%) \mathrm{b}$ & $0.79 \pm 0.02(4.49 \%) \mathrm{a}$ \\
& Oleic acid (C18:1) & $3.62 \pm 0.13(18.09 \%) \mathrm{b}$ & $6.97 \pm 0.22(30.82 \%) \mathrm{a}$ & $2.71 \pm 0.12(15.39 \%) \mathrm{c}$ \\
& Linoleic acid (C18:2) & $9.54 \pm 0.98(47.61 \%) \mathrm{a}$ & $9.10 \pm 0.13(40.24 \%) \mathrm{a}$ & $8.27 \pm 0.13(47.00 \%) \mathrm{b}$ \\
& Linolenic acid (C18:3) & $4.84 \pm 0.07(24.16 \%) \mathrm{a}$ & $4.50 \pm 0.04(19.91 \%) \mathrm{b}$ & $4.45 \pm 0.07(25.31 \%) \mathrm{b}$ \\
& Total & $20.4 \pm 1.29(100 \%)$ & $22.62 \pm 0.46(100 \%)$ & $17.59 \pm 0.41(100 \%)$ \\
Carotenoid & $10.31 \pm 0.45 \mathrm{a}$ & $9.87 \pm 0.34 \mathrm{a}$ & $9.87 \pm 0.45 \mathrm{a}$ \\
& Lutein & $1.14 \pm 0.06 \mathrm{~b}$ & $1.49 \pm 0.06 \mathrm{a}$ & $1.48 \pm 0.07 \mathrm{a}$ \\
& Zeaxanthin & $11.44 \pm 0.52 \mathrm{a}$ & $11.365 \pm 0.39 \mathrm{a}$ & $11.35 \pm 0.52 \mathrm{a}$ \\
& Total & $119.76 \pm 3.15 \mathrm{a}$ & $\mathrm{ND} \mathrm{b}^{\mathrm{y}} \mathrm{b}$ & $\mathrm{NDb}$ \\
& Myricetin & $121.60 \pm 4.56 \mathrm{a}$ & $\mathrm{NDb}$ & $\mathrm{NDb}$ \\
& Luteolin & $249.88 \pm 8.19 \mathrm{a}$ & $113.69 \pm 6.25 \mathrm{c}$ & $218.64 \pm 9.38 \mathrm{~b}$ \\
& Kaempferol & $491.24 \pm 14.91 \mathrm{a}$ & $111.69 \pm 6.25 \mathrm{c}$ & $218.64 \pm 9.38 \mathrm{~b}$ \\
& Total & $166.05 \pm 5.19 \mathrm{a}$ & $5.75 \pm 0.18 \mathrm{c}$ & $77.65 \pm 2.10 \mathrm{~b}$ \\
& Delphinidin & $111.08 \pm 2.60 \mathrm{a}$ & $2.22 \pm 0.27 \mathrm{~b}$ & $113.48 \pm 2.04 \mathrm{a}$ \\
& Cyanidin & $277.13 \pm 8.072 \mathrm{a}$ & $7.98 \pm 0.34 \mathrm{c}$ & $191.13 \pm 0.14 \mathrm{~b}$ \\
\hline
\end{tabular}

${ }^{\mathrm{z})}$ Different letters represent significant differences among means of lentil seed varieties $(P<0.05)$.

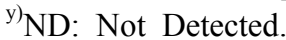



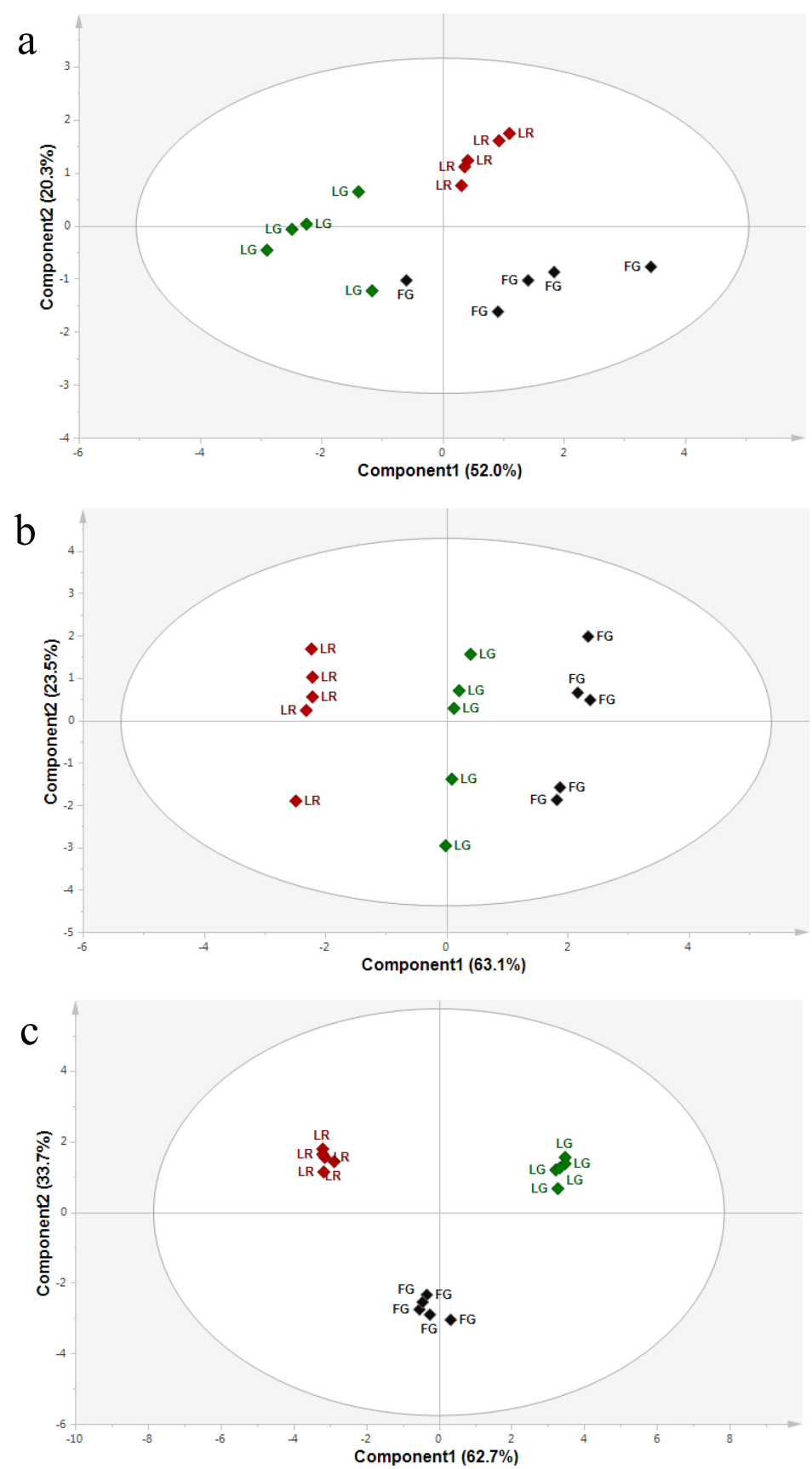

Fig. 3. PCA score plots obtained from the three lentil varieties. (a) Fatty acids, (b) phytochemicals, (c) phenolic acids.

suggest that the profiles of fatty acid, especially the unsaturated fatty acids were diverse or different according to the color profiles.

\section{Carotenoid, flavonoid, and anthocyanin}

All carotenoids including flavonoids, and anthocyanins were analysed by HPLC, on the basis of which the metabolic differences among the three genotypes and the relationships with their colors were analyzed. Carotenoids, which are fat-soluble pigments, mainly confer yellow, orange, and red colors to plants and play an important role in human nutrition as precursors of vitamin A and antioxidants, of which lutein and zeaxanthin are important for eye health and protect cells from carcinogens (Wang et al. 


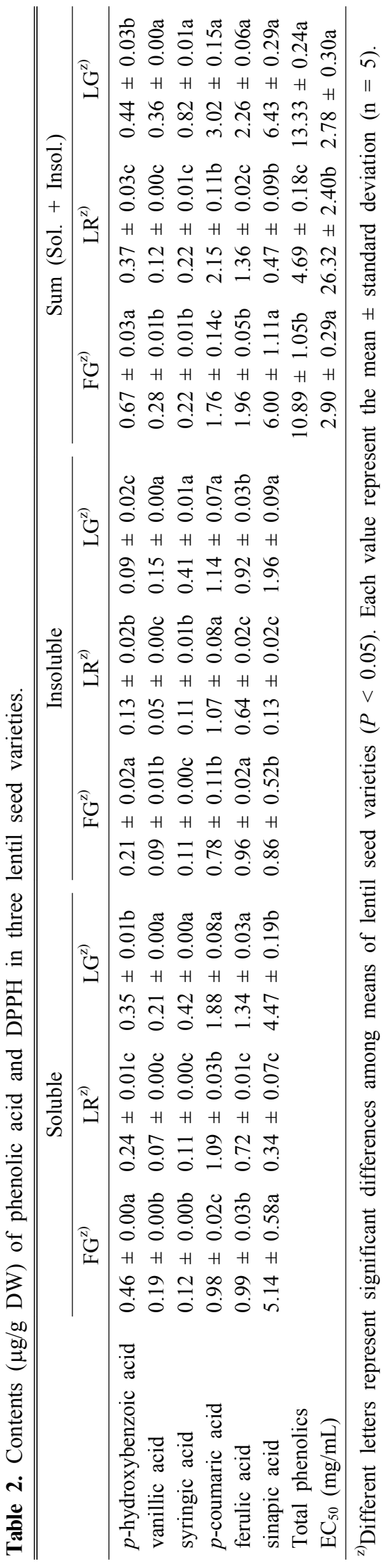

2006). Only lutein and zeaxanthin were identified in the present study (Table 1). The levels of lutein did not significantly vary among FG, LR, and LG $(P>0.05)$, and were 6-9 times higher than those of zeaxanthin. These results were consistent with the report of Zhang et al. (2014). The zeaxanthin content of FG was significantly lower than those of LR and LG $(P<0.05)$, with the highest mean contents noted for LR followed by LG and FG. This result was similar to that of Zhang et al. (2014), in which the average zeaxanthin level was higher in red than in green lentils.

Flavonoids are vacuolar water-soluble pigments that are classified into flavonols, flavones, flavanones, catechins, anthocyanins, and chalcones (Kim et al. 2013). In the three varieties, we identified flavonoids and anthocyanins, which are presented in Table 1. Myricetin and luteolin flavonoids were detected in FG but not in LR and LG. Similarly, in a study of Xu and Chang (2010) on 11 lentils grown in the northern United States, the luteolin content of French green lentils was higher than those of other lentil varieties. Thus, it can be suggested that lentils showing relatively dark colors are higher in luteolin than red or light green lentils. Kaempferol levels were significantly different among FG, LR, and LG $(P<0.05)$, with the highest noted for FG. Cyanidin is reportedly the major anthocyanin in black soybean seed coats (Choung et al. 2001). Delphinidin and cyanidin anthocyanins were detected in all varieties, and they also showed significant differences depending on variety $(\mathrm{FG}>\mathrm{LG}>\mathrm{LR})(P<0.05)$. PCA of the carotenoid, flavonoid, and anthocyanin data showed a clear discrimination by color (Fig. 3b). The loading plots contributed mainly to PC1 construction, representing $63.1 \%$ of the total variability. These results suggested that metabolites related to seed color genotype were clearly distinguishable, indicating nutritional differences.

\section{Phenolic acids}

To estimate the anti-oxidative capacity of the three lentil varieties, contents of six phenolic acids ( $p$-hydroxybenzoic acid, vanillic acid, syringic acid, $p$-coumaric acid, ferulic acid, and sinapic acid) that have anti-oxidative effects were measured by using GC-TOF MS (Table 2). The phenolic compounds were divided into methanol-soluble and 
-insoluble forms for analysis. The major phenolic acids in all three varieties were coumaric acid, ferulic acid, and sinapic acid. All six phenolic acids of both soluble and insoluble forms in the three lentil varieties showed significant differences among varieties $(P<0.05)$. Except for coumaric acid, all phenolic acids were higher in FG and LG than in LR $(P<0.05)$. Soluble sinapic acids were the highest in FG and LG, in agreement with the report by $\mathrm{Xu}$ and Chang (2010). The effectiveness of plant phenolic compounds as antioxidants is generally diverse; however, this does not always depend on their quantities, but may well be dictated by the chemical structures of their constituents (Shahidi and Naczk 2004; Albishi et al. 2013). Total phenolic acid contents (soluble and insoluble) of three varieties were clearly distinguishable by variety in PCA, and the loading plots of phenolic acids contributed mainly to PC1 construction, representing $62.7 \%$ of the total variability (Fig. 3c). These results suggested that different colors relate to different anti-oxidant nutrient profiles. In previous studies of colored plants, including cauliflower (purple, green $>$ white, yellow), small colored potatoes (colored $>$ regular), soybeans (black $>$ yellow), and sweet potatoes (purple $>$ white, orange), the contents of phenolic acid in the colored plants were higher than in the non-colored plants and there were significant differences among the colored plants (Park et al. 2013; Lee et al. 2014; Park et al. 2014; Park et al. 2016).

\section{Antioxidant activity}

DPPH radical scavenging activity of extracts was measured to investigate the antioxidant activity of the three lentil varieties. BHT, a synthetic oxidizer, was used as a control. Based on measurements of the optical density of the reaction mixture, the $\mathrm{EC}_{50}$, which is the concentration of sample required to inhibit $50 \%$ of DPPH radicals, was calculated, with lower $\mathrm{EC}_{50}$ indicating higher antioxidant activity (Table 2). The $\mathrm{EC}_{50}$ values were within the range of 2.78-6.32 mg/mL DW. DPPH activities of FG and LG were $2.9 \mathrm{mg} / \mathrm{mL}$ DW and $2.78 \mathrm{mg} / \mathrm{mL} \mathrm{DW}$, respectively, and were stronger than that of LR. The DPPH assay confirmed that lentil extracts with higher phenolic and flavonoid contents had stronger antiradical action (Alshikh et al. 2015). These results were consistent with those of flavonoid, anthocyanin, and phenolic acid contents, suggesting that antioxidant activity is also related to color-related metabolites, especially for flavonoids and anthocyanins. Consistent with the study of Xu and Chang (2010), LR showed relatively low antioxidant activity, while the green and yellow lentils showed high antioxidant activities (LR $<$ FG $<$ LG, red $<$ dark green $<$ light green).

\section{Correlation analysis and the HCA of metabolites}

Correlation analysis and the HCA were performed to examine the relationships among metabolites analyzed from different colored lentils (Fig. 4). The result of HCA could illustrated the extent of distances among compositional nutrients or among lentil varieties, respectively. Applied variables were based on their mutual correlation coefficients from SAS analysis. Distances between samples are typically displayed in a dendrogram which provides a simple graphical view of sample groupings; the length of the branches (cluster distance) is a measure of the degree of similarity between metabolites or groups of samples. The longer distance showed in the dendrogram meant the lower correlation between analyzed variables (Fig. 4). In the horizontal line listed with analyzed metabolites, the correlation value (r) between asparagine and glucose was 0.10617 , which were lowest in the result of correlation analysis. This means that these two variables are less influenced by each other. The correlations between myristic acid and succinic acid $(r=0.49927)$ and between inositol and isolecine $(r=0.41493)$ were also low, in order, showing relatively longer distances in the result of HCA dendrogram (Fig. 4). In the vertical dendrogram, the distances between FG and LG, between LG and LR, and between FG and LR were longer showing lower correlation values than those between variables in the same varieties showing higher correlation values. It could be demonstrated that the lentils with different colors by different color varieties. These results may also offer opportunities for further omics studies related to the safety assessment of new biotechnology plants. 


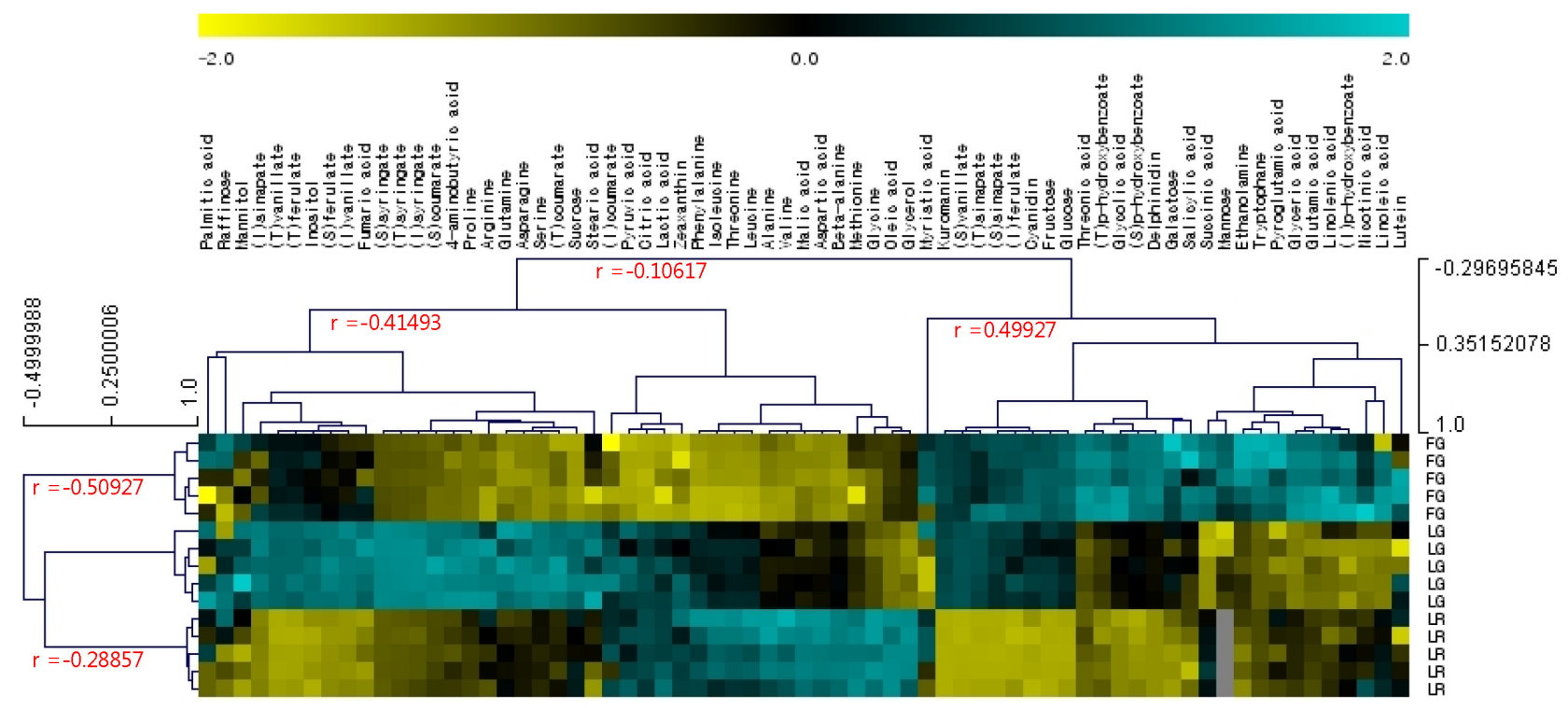

Fig. 4. Correlation matrix and cluster analysis of metabolites obtained from seed of the three lentil varieties. Each square indicates the Pearson's correlation coefficient of a pair of compounds, and the value for the correlation coefficient is represented by the intensity of the blue or red color, as indicated on the color scale.

\section{DISCUSSION}

This study was aimed to compare the metabolite profiles related to antioxidant components in the various lentils with different colors by investigating primary and derivative secondary metabolites. Among 42 hydrophilic low molecular weight compounds, shikimic acid and trehalose were found only in the FG and LG variety, respectively. Mannose was found exclusively in the FG and LG varieties. In the fatty acid contents, LR variety showed highest contents, which was contrary result to the phenolics and DPPH activity. Three kind of lentils were not different statistically with each other in the lutein $(P>0.05)$ which was 6-9 times higher than zeaxanthin. Myricetin and luteolin were detected only in the FG, and these were not detected in the LR and LG. In addition, there were significant differences in kaempferol, delphinidin and cyanidin among three varieties (FG $>$ LG $>$ LR). The phenolic acids $p$-hydroxybenzoic acid, vanillic acid, syringic acid, $p$-coumaric acid, ferulic acid, and sinapic acid, in both soluble and insoluble forms, were significantly different among the three varieties. The levels of all phenolic acids except coumaric acid were significantly higher in FG and LG than in LR. FG and LG varieties had stronger radical scavenging activities than LR in the result of DPPH radical scavenging assay. The antioxidative activity is difficult to detect because of its complex nature of phytochemicals. It is necessary to apply multiple assay to evaluate the activities of plant phytochemicals. Because DPPH radical is a stable free radical, it has been extensively used for identifying the activities of plant varieties. In this study, the phenolic contents and DPPH radical scavenging activities were analyzed to compare the antioxidant function among different colored lentils, and their results were showed consistently (LR < $\mathrm{FG}<\mathrm{LG}$ ). It was reported that the antioxidant activity of plant extracts could be attributed to the total phenolic content in the previous research (Zou et al. 2011). The present results were similar to the research of Zou et al. (2011) showing the antioxidant activities are highly related to colors, which is the lentils with higher contents in the phenolics showed the higher contents or activities in the flavonoids, anthocyanins and antioxidant properties. All multivariate analysis showed similar classification of various lentils with different colors, which meant that metabolic profiling using chemometric tools could discriminate between plants with different genotype characteristics. In conclusion, the profiles of lentils with 
different colors were different in the anti-oxidative metabolites such as carotenoids, anthocyanins, and phenolic acids. It was possible to identify meaningful compositional differences and relationships between metabolites related to colors in lentil varieties with PCA, Pearson correlation, and HCA analyses.

\section{ACKNOWLEDGEMENTS}

This study was supported by the National Institute of Agricultural Science (Code PJ01175201), Rural Development Administration, Republic of Korea. We are also thankful to the reviewers whose comments led to substantial improvements in this paper and to the Executive Editor for his consideration.

\section{REFERENCES}

Albishi T, John JA, Al-Khalifa AS, Shahidi F. 2013. Antioxidative phenolic constituents of skins of onion varieties and their activities. J. Funct. Foods. 5: 1191-12203.

Alshikh N, De Camargo AC, Shahidi F. 2015. Phenolic of selected lentil cultivars: Antioxidant activities and inhibition of low-density lipoprotein and DNA damage. J. Funct. Foods. 18: 1022-1038.

AOCS. 1997. Trypsin inhibitor activity. In official methods and recommended practices of the American Oil Chemists Siciety. Method Ba.12-75. Champaign IL.

Bhakta D, Siva R. 2010. Determination of lycopene and its antioxidant activities in Indian tomatoes. J. Pharm. Res. 3: 933-937.

Bouvier F, Isner JC, Dogbo O, Camara B. 2005. Oxidative tailoring of carotenoids: a prospect towards novel functions in plants. Trends Plant Sci. 10: 187-194.

Choung MG, Baek IY, Kang ST, Han WY, Shin DC. 2001. Isolation and determination of anthocyanins in seed coats of black soybean (Glycine man (L.) Merr.). J. Agr. Food Chem. 49: 5848-5851.

Cazzonelli CI. 2011. Carotenoids in nature: insights from plants and beyond. Funct. Plant Biol. 3: 833-847.

Dueñas M, Hernández T, Estrella I. 2002. Phenolic composition of the cotyledon and the seed coat of lentils (Lens culinaris L.). Eur. Food Res. Technol. 215: 478-483.

Harborne JB. 1993. Biochemical interactions between higher plants. Introduction to Ecological Biochemistry. 4rd (ed) Academic press London. pp. 243-263.

Jaakola L, Hohtola A. 2010. Effect of latitude on flavonoid biosynthesis in plants. Plant Cell Environ. 33: 12391247.

Kang MH, Oh MK, Bang JK, Kim DH, Kang CH, Lee BH. 2000. Varietal difference of lignan contents and fatty acids composition in Korean sesame cultivars. Korean J. Crop Sci. 45: 203-206.

Kim YH, Lee JH, Lee YS, Yun HT. 2006. Antioxidant activity and extraction efficiency of anthocyanin pigments in black colored soybean. Korea Soybean Digest. 23: 1-9.

Kim SL, Lee YH, Chi HY, Lee SJ, Kim SJ. 2007. Diversity in lipid contents and fatty acid composition of soybean seeds cultivated in Korea. Korean J. Crop Sci. 52: 348-357.

Kim JK, Lee SY, Chu SM, Lim SH, Suh SC, Lee YT, et al. 2010. Variation and correlation analysis of flavonoids and carotenoids in Korean pigmented rice (Oryza sativa L.) cultivars. J. Agr. Food Chem. 58: 12804-12809.

Kim YB, Park SY, Thwe AA, Seo JM, Suzuki T, Kim SJ, et al. 2013. Metabolomic analysis differential expression of anthocyanin biosynthetic genes in white- and red-flowered buckwheat cultivars (Fagopyrum esculentum). J. Agr. Food Chem. 61: 10525-10533.

Kim HS, Ahn JJ, Choi TH, Hwang TY. 2014. Screening of DPPH radical scavenging and antimicrobial activity of extracts from local some native plants. Korean J. Food Preserv. 21: 593-599.

Lee LS, Choi EJ, Kim CH, Kim YB, Park JD. 2014. Quality characteristics and antioxidant properties of black and yellow soybeans. Korean J. Food Sci. Technol. 46: 757-761.

Mira NVM, Massaretto IL, Pascual CSCI, Marquez UML. 2009. Comparative study of phenolic compounds in different Brazilian rice (Oryza sativa, L.) genotypes. J. Food Compos. Anal. 22: 405-409.

Nithiyanantham S, Selvakumar S, Siddhuraju P. 2012. Total phenolic content and antioxidant activity of two different solvent extracts from raw and processed legumes, Cicerarietinum L. and Pisumsativum L. J. Food Compos. Anal. 27: 52-60.

Oh YJ, Cho SK, Kim KH, Kim YJ, Kim TS, Kim JG, et al. 
2009. A new soybean cultivar, "Galchae" for sprout with brown seed coat, small seed size and high sprout yielding. Korean J. Breed Sci. 41: 324-327.

Park SJ, Kwon MS, Shin KY, Rha YA. 2014. Comparison of nutritional components and physicochemical properties of small colored potatoes and small regular potatoes. Korean J. Culinary Research. 20: 80-89.

Park SY, Lim SH, Ha SH, Yeo YS, Park WT, Kwon DY, et al. 2013. Metabolite profiling approach reveals the interface of primary and secondary metabolism in colored cauliflowers (Brassica oleracea L. ssp. botrytis). J. Agric. Food Chem. 61: 6999-7007.

Park SY, Lee SY, Yang JW, Lee JS, Oh SD, Oh SW, et al. 2016. Comparative analysis of phytochemicals and polar metabolites from colored sweet potato (Ipomoea batatas L.) tubers. Food Technol. Biotech. 25: 283-291.

Shahidi F, Naczk M. 2004. Phenolics in food and nutraceuticals. CRC Press Inc. pp. 17-83.

Shahidi F, Zhong Y. 2008. Bioactive peptides. J. AOAC Int. 91: 914-931.

Steuer R. 2006. Review: on the analysis and interpretation of correlations in metabolomic data. Brief Bioinform.7:
151-158.

Wang M, Tsao R, Zhang S, Dong Z, Yang R, Gong J, et al. 2006. Antioxidant activity, mutagenicity/anti-mutagenicity, and clastogenicity/anti-clastogenicity of lutein from marigold flowers. Food Chem. Toxicol. 44: 1522-1529.

$\mathrm{Xu}$ B, Chang SKC. 2010. Phenolic substance characterization and chemical and cell-based antioxidant activities of 11 lentils grown in the northern United States. J. Agric. Food Chem. 58: 1509-1517.

Yoon TH, Im KJ, Kim DH. 1984. Fatty acid composition of lipids obtained from Korean soybean varieties. Korean J. Food Sci. Technol. 16: 375-382.

Zhang B, Deng Z, Tang Y, Chen P, Liu R, Ramdath DD, et al. 2014. Fatty acid, carotenoid and tocopherol compositions of 20 Canadian lentil cultivars and synergistic contribution to antioxidant activities. Food Chem. 161: 296-304.

Zou Y, Chang SKC, Gu Y, Qian SY. 2011. Antioxidant activity and phenolic compositions of lentils (Lens culinaris var. Morton) extract and its fractions. J. Agric. Food Chem. 59: 2268-2276. 